\title{
Bioavailability and Dissolution Rate Enhancement of an Anticancer Drug Bicalutamide by Encapsulation into Mesoporous Silica Nanoparticles: Effects of Amine Functionalization and Caco-2 Cell Permeability Study
}

\author{
Seema Saroj, Sadhana Rajput* \\ Department of Pharmaceutical Quality Assurance, Centre for Excellence in NDDS, Faculty of Pharmacy, G.H. Patel Pharmacy Building, \\ The Maharaja Sayajirao University of Baroda, Vadodara, Gujarat, INDIA.
}

\begin{abstract}
Background: The present study was undertaken with the aim of dissolution rate and Bioavailability enhancement of anticancer drug Bicalutamide (BIC) via encapsulation into mesoporous silica nanoparticles (MSNs). Materials and Methods: The bare (MCM-41) and surface decorated MSNs were subjected to thorough characterization pre and post drug loading by Fourier transform infrared spectroscopy, Nitrogen sorption analysis, Differential Scanning Calorimetry, Thermogravimetric analysis, Small angle and wide angle $\mathrm{X}$-ray diffraction, Scanning electron microscopy and Transmission electron microscopy. Introduction of Nitrogen post functionalization was confirmed by the elemental analysis. Synthesized MSNs were surface decorated with amine groups (AMN-MSN) and in-depth study of effect of functionalization and food on dissolution kinetics was done. Developed MSNs and AMN-MSNs were thoroughly characterized pre and post BIC loading. In vitro intestinal permeability study was performed to determine the apparent permeability of synthesized MSNs. The formulations were also investigated for their in vivo behaviour by oral administration to male Swiss albino mice and various pharmacokinetic parameters calculated. Results: A significant increase in permeability was obtained the dissolution rate and bioavailability were found to be 3.12 and 2.71 times enhanced in MCM-41 as comparison to the BIC suspension. Conclusion: Thus, MSNs could serve as an effective platform for delivering of various anti-cancer drugs and enhancing their efficiency.

Key words: Bicalutamide, Functionalized Mesoporous silica nanoparticles, Dissolution, Caco-2 cells permeability, Bioavailability.
\end{abstract}

\section{INTRODUCTION}

About more than 40 percent (\%) of market approved drugs and $90 \%$ of drugs currently in the discovery pipeline face solubility issues. ${ }^{1}$ The solubility limitations can act as a major barrier for bioavailability of drugs. Low bioavailability thus limits the effectiveness of various therapeutic moieties. Various drugs facing solubility and bioavailability issues have been successfully formulated into drug products with improved dissolution and enhanced bioavailability.
Bicalutamide (BIC) is a non-steroidal anti-androgen agent widely used in the effective treatment of prostate cancer. ${ }^{2}$ It is chemically N-[-4-cyano-3-(trifluoromethyl) phenyl]-3-[(4-fluorophenyl)sulfnyl]-2hydroxy-2-methylpropanamide. ${ }^{3}$ It belongs to Biopharmaceutical classification class (BCS) Class-II and hence its efficacy is limited by solubility constraints, which in turn limits the bioavailability as well. Therefore, it becomes imperative to investigate novel
Submission Date: 12-01-2020; Revision Date: 20-04-2020; Accepted Date: 26-06-2020

DOI: 10.5530/ijper.54.3.108 Correspondence:

Prof. Sadhana Rajput Department of Pharmaceutical Quality Assurance, Centre for Excellence in NDDS, Faculty of Pharmacy, G.H. Patel Pharmacy Building, The Maharaja Sayajirao University of Baroda, Vadodara-390002, Gujarat, INDIA.

Phone: +91-0265-2434187 E-mail: sjrajput@gmail.com

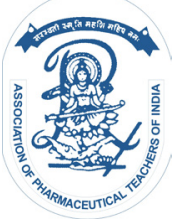

www.ijper.org 
drug delivery systems for enhancing the dissolution rate and bioavailability of this widely used anticancer drug. So far, different types of delivery systems have been investigated for dissolution and bioavailability enhancement of BIC including nanocrystals, ${ }^{3}$ Solid dispersions, ${ }^{4,5}$ Nano dispersion, ${ }^{6}$ inclusion complex ${ }^{7}$ and adsorption phenomena. ${ }^{8}$ However, till date there are no reports employing the use of mesoporous silica nanoparticles for investigating dissolution and bioavailability enhancement of BIC. It was thought of interest and great use to study the success rate and usefulness of MSNs in this regard. This provided a motivation and base to perform the entire study. MSNs have various advantages over other Nano systems like well-defined surface properties providing ease of functionalization and various functional group attachment, ${ }^{9}$ low in vivo toxic profile with good biocompatibility, ${ }^{10}$ zero premature leakage, stability, high pore volume and surface area, ${ }^{11}$ versatility and biodegradability. ${ }^{12,13}$ These all features combined contribute to their versatility and effectiveness as drug delivery nanocarriers.

In the present research work, investigating effect of bare MCM-41 and functionalized nanocarriers on dissolution and bioavailability; promising results were obtained with visible increments obtained in both. The effect of food on formulation release was studied in different biorelevant media imitating fed and fasted state gastrointestinal conditions. The dissolution kinetics for release study was studied by fitting the $\%$ cumulative release data to various kinetic models. The dissolution study and pharmacokinetic studies performed in male Swiss albino mice (SAM) exhibited commendable results with 3.12 and 2.61 times increment in dissolution rate and bioavailability respectively. The outcomes of the present research exhibits that MSNs can be used as potent and safe vehicles for delivery of drugs possessing solubility and bioavailability barriers in way of their therapeutic effectiveness. The exploration of various technologies including MSNs would result in improved formulations leading to better therapeutic applications with improved patient compliance.

\section{MATERIALS AND METHODS}

\section{Materials}

Pure Active pharmaceutical ingredient (API) Bicalutamide (BIC) was obtained as a sample gratis from Intas Pharmaceuticals (Ahmedabad, Gujarat, India). Chemicals and reagents used in synthesis like Fumed silica, tetramethyl ammonium hydroxide pentahydrate (TMAOH, purity $\geq 98 \%$ ) and (3-Aminopropyl) triethoxy silane APTES (99\% purity) used in surface functionalization was procured from Sigma Aldrich (St Louis, USA). surfactant Cetyl trimethyl ammonium bromide (CTAB) was obtained from Lobachemie (Mumbai, India). Chemicals or constituents of the dissolution media like Hydrochloric acid, acetic acid, monobasic potassium phosphate, potassium dihydrogen phosphate, ammonium acetate and sodium chloride were purchased from S.D. Fine Chem Ltd. Vital ingredients like pancreatin, pepsin, sodium taurocholate and Lecithin were obtained from Sigma Aldrich. Toluene and analytical grade methanol were bought from Fischer scientific (Mumbai, India). De-ionized water was used in entire study unless mentioned otherwise. Caco-2 cells were purchased from National centre for cell sciences (NCCS, Pune, India). Media used in cell line studies DMEM, Trypsin-EDTA and FBS, cell culture flasks were obtained from HI media Laboratories. Transwell inserts (Nunc, Denmark) and Lucifer yellow dye was procured from Thermo Scientific India. MTT (3-(4,5-dimethylthiazol-2-yl)-2,5-diphenyltetrazolium bromide) dye was purchased from Sigma Aldrich.

\section{METHODS}

\section{Synthesis of MCM-41 type nanoparticles}

MCM-41 type MSNs were synthesised based on a template based synthesis method detailed in the literature adopted with slight moderation. ${ }^{14}$ Precisely, 2.4g CTAB was added to 33 millilitres $(\mathrm{mL})$ of deionized water and blended for $5 \mathrm{~min}$ (min). To this, $3.25 \mathrm{~mL}$ of TMAOH was added drop wise under non-stop stirring. This was followed by addition of $3 \mathrm{~g}$ fumed silica after $10 \mathrm{~min}$. Continuous stirring was provided to the reaction mixture for $2 \mathrm{~h}$. Thereafter, hydrothermal treatment was given for $48 \mathrm{~h}$. The slurry obtained was filtered, washed and dried. The fine powder obtained was subjected to calcination at $823 \mathrm{~K}$ for $5 \mathrm{~h}$. The final product obtained was labelled as MCM-41.

\section{Amine functionalization of MCM-41}

Post synthetic functionalization strategy was employed for grafting amino groups onto MCM-41 MSNs. ${ }^{15}$ Accurately weighed $500 \mathrm{mg}$ MCM-41 was transferred to $100 \mathrm{~mL}$ Round bottom flask (RBF). Followed by addition of $50 \mathrm{~mL}$ toluene and $6.87 \mathrm{~mL}$ of APTES. The reaction mixture was heated to a higher temperature of $70^{\circ} \mathrm{C}$ for $12 \mathrm{~h}$. Thereafter it was allowed to cool to room temperature, with subsequent filtration and washing with methanol. The synthesized carrier was tagged as MCM-41-AMN. The grafting of amine group 


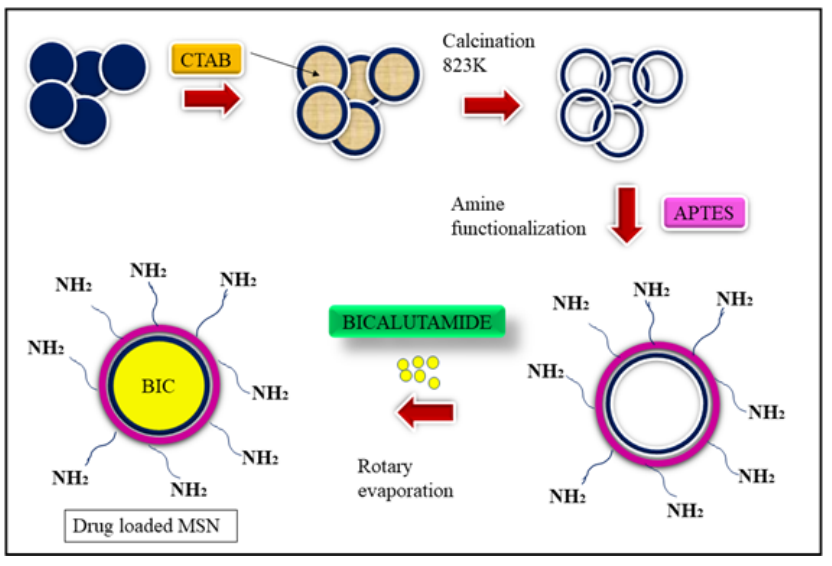

Figure 1: Schematic representation of synthesis of MSNs, their functionalization and BIC loading into the mesopore.

was confirmed by FT-IR Spectroscopy. The entire synthesis scheme is depicted in Figure 1.

\section{Drug entrapment and loading into synthesized carriers}

BIC was selected as a model anticancer drug and loaded into mesopores by solvent rotary evaporation method. Drug solution was prepared in methanol. Mesoporous carrier was added to this and stirred continuously for $2 \mathrm{hr}$. The drug and carrier ratio taken was 1:1.5 respectively. This was followed by evaporation of volatile solvent at $70^{\circ} \mathrm{C}$. Complete drug loading into the mesopores was confirmed by DSC, FT-IR and WXRD. Loading and entrapment efficiency were calculated by the formulas given below.

$$
\begin{aligned}
& \text { Loading efficiency }(\%)=\frac{\text { Wt of drug BIC in MSNs }(\mathrm{mg})}{\text { Initial wt of BIC }(\mathrm{mg})} \\
& \text { Entrapment efficiency }(\%)=\frac{\text { Wt of drug BIC in MSNs }(\mathrm{mg})}{\text { Initial wt of BIC loaded MSNs }(\mathrm{mg})}
\end{aligned}
$$

\section{Solid state characterization}

Butchi ${ }^{\mathrm{TM}}$ Rotary evaporator was used for evaporation of solvent during drug loading. Synthesized nanoparticles were investigated for their morphology and mesoporous structural integrity by SEM (FEIQ uanta 200 model, Thermo Scientific). And TEM (TEM-200, Phillips, India) analysis operated at $200 \mathrm{kv}$ voltage and $2.4 \AA$ resolution. Bruker ALPHA-T instrument was used to record the FT-IR spectra of nanoparticles and drug. Melting point was determined by SHIMADZU TA-60 DSC instrument operated at a ramp rate of $10^{\circ} \mathrm{C} / \mathrm{min}$. The $\%$ weight loss was determined by TGA analysis (SHIMADZU TGA Analyzer $50 \mathrm{WS}$ ) on a $5 \mathrm{mg}$ sample with ramp rate of $10^{\circ} \mathrm{C} / \mathrm{min}$ and investigation temperature range as $25-700^{\circ} \mathrm{C}$. Zeta potential and size of MSNs was determined by Dynamic light scattering
(DLS) using Malvern Zetasizer Nano ZS90 instrument. Small angle XRD measurements were carried out on EMPYREAN, PAN alytical model equipped with $\mathrm{Cu}$ $\mathrm{K}$ radiation beam and operating at $40 \mathrm{kV}$ and $30 \mathrm{kV}$ to determine characteristic peaks unique to MCM-41. Crystalline nature of BIC and absence of this post entrapment into mesoporous core was confirmed by Wide Angle XRD spectra taken on Bruker AXS instrument with D8 Focus software installed. Surface area and porosity measurements were carried out by Nitrogen adsorption-desorption isotherm analysis using ASAP 2020 apparatus from micromeritics (Norcross, USA) temperature of $-196^{\circ} \mathrm{C}$. Before proceeding with analysis, the samples were degassed under vacuum for $5 \mathrm{~h}$ at $70^{\circ} \mathrm{C}$ in the degas port of adsorption analyser.

\section{Dissolution study}

Veego USP type II paddle apparatus was used to perform the dissolution study in $1000 \mathrm{~mL}$ dissolution media at $50 \mathrm{rpm}$ maintaining $37 \pm 0.5^{\circ} \mathrm{C}$. The in vitro dissolution study was performed for BIC, Marketed Formulation (MF), BIC-MCM-41 and BIC-MCM-41-AMN in various media viz water (with addition of $0.5 \%$ SLS), FaSSGF, FeSSGF, FaSSIF and FeSSIF (Fast and Fed state biorelevant media) to study the drug release pattern from non-functionalized and functionalized mesoporous systems in presence and absence of food. The composition of various dissolution media used is stated in Table 1. The composition of tablets prepared having $50 \mathrm{mg}$ BIC tablets is given in Table 2. Samples were withdrawn at regular intervals and analysed by UV spectrophotometry at wavelength maxima of $272 \mathrm{~nm}$. The data obtained was fitted to various kinetic models and best fit was determined.

\section{MTT assay on Caco-2 cells}

For the purpose of studying cytotoxicity of BIC, BIC-MCM-41 and BIC-MCM-41-AMN MSNs. MTT assay was carried out on Caco-2 cells. Caco-2 cells were cultured at low passage number. The cells were maintained at $37^{\circ} \mathrm{C}$ in an incubator and $5 \%$ Carbon dioxide supply in DMEM medium supplemented with $20 \%$ FBS and 1\% Pen-strep solution to aid in avoiding any sort of contamination. The cells were seeded at a density of 10,000 cells per well in complete DMEM media in 96 well microtiter plates. This was followed by nanoparticle treatment for 24 and $72 \mathrm{~h} \mathrm{n}$ fresh incomplete DMEM medium in concentration range $0.1-100 \mu \mathrm{g} / \mathrm{mL}$. Next, $100 \mu \mathrm{L}$ MTT dye was added to each well and incubated for $4 \mathrm{hr}$. Ultimately, $100 \mu \mathrm{L}$ DMSO was added to solubilize the formazan formed. After elapsing of $10 \mathrm{~min}$ the plates were read at $590 \mathrm{~nm}$ 
Table 1: Composition of various dissolution media used. (USP official MEDIA = Water ( $0.5 \%)$, Fast state simulated gastric fluid (FaSSGF), Fed state simulated gastric fluid (FeSSGF), Fast state simulated intestinal fluid (FaSSIF), Fed state simulated intestinal fluid (FeSSIF)).

\begin{tabular}{|c|c|c|c|c|c|c|}
\hline Sr No. & COMPOSITION & Official media & FaSSGF & FeSSGF & FasSIF & FeSSIF \\
\hline 1 & Lecithin & - & $20 \mu \mathrm{M}$ & - & $0.75 \mathrm{mM}$ & $3.75 \mathrm{mM}$ \\
\hline 2 & Sodium taurocholate & - & $80 \mu \mathrm{M}$ & - & $3 \mathrm{mM}$ & $15 \mathrm{mM}$ \\
\hline 3 & Pepsin & - & $0.1 \mathrm{~g}$ & - & - & - \\
\hline 4 & Acetic acid & - & - & $2.1 \mathrm{~g}$ & - & $8.65 \mathrm{~g}$ \\
\hline 5 & Sodium acetate & - & - & $4.01 \mathrm{~g}$ & - & \\
\hline 6 & Sodium chloride & - & $1 \mathrm{~g}$ & $13.85 \mathrm{~g}$ & $3.093 \mathrm{~g}$ & $11.87 \mathrm{~g}$ \\
\hline 7 & Sodium Lauryl Sulphate & $0 . .5 \%$ & $0 . .5 \%$ & $0 . .5 \%$ & $0 . .5 \%$ & $0 . .5 \%$ \\
\hline 8 & Milk & - & - & $500 \mathrm{~mL}$ & - & - \\
\hline 9 & Pancreatin & - & - & - & - & - \\
\hline 10 & Monobasic potassium phosphate & - & - & - & $1.977 \mathrm{~g}$ & - \\
\hline 11 & Sodium hydroxide & - & - & - & $0.174 \mathrm{~g}$ & $4.04 \mathrm{~g}$ \\
\hline 12 & $\mathrm{pH}$ & 7.2 & 1.6 & 1.6 & 6.5 & 6.5 \\
\hline 13 & Deionized Water & Upto $1000 \mathrm{~mL}$ & Upto $1000 \mathrm{~mL}$ & Upto $1000 \mathrm{~mL}$ & Upto $500 \mathrm{~mL}$ & Upto $1000 \mathrm{~mL}$ \\
\hline
\end{tabular}

Table 2: Excipients used in tablet preparation.

\begin{tabular}{|c|c|c|c|}
\hline $\begin{array}{l}\text { Sr } \\
\text { No. }\end{array}$ & Excipient Name & Use & Amount \\
\hline 1 & $\begin{array}{l}\text { Sodium starch } \\
\text { glycolate }\end{array}$ & Disintegrating agent & $2-5 \%$ \\
\hline 2 & $\begin{array}{l}\text { Polyvinyl } \\
\text { pyrrolidone }\end{array}$ & Binder & $2-4 \%$ \\
\hline 3 & Talc & Glidant & $0.5-1 \%$ \\
\hline 4 & $\begin{array}{l}\text { Magnesium } \\
\text { stearate }\end{array}$ & Lubricant, diluent & $0.4-1 \%$ \\
\hline 5 & $\begin{array}{l}\text { Microcrystalline } \\
\text { cellulose }\end{array}$ & $\begin{array}{l}\text { Disintegrating agent in } \\
\text { Direct compression }\end{array}$ & q.s. \\
\hline
\end{tabular}

using plate reader, \% cell viability was calculated as per the formula given below.

$\%$ Cell visibility $=\frac{O \cdot D_{\text {sample }}-O \cdot D_{\text {blank }}}{O . D_{\text {negative control }}-O . D_{\text {blank }}} \times 100$

Where, Blankwas devoid of any cells.

O.D. stands for optical density.

\section{Caco-2 permeability study}

Caco-2 cell line was procured from NCCS-Pune and maintained at $5 \%$ Carbon dioxide, $37^{\circ} \mathrm{C}$ and complete RPMI-1640 with 20\% FBS and 1\% antibiotics (Pen-
Strep solution). Lucifer yellow was used to check the membrane integrity. The Caco-2 cells were grown on Trans well inserts having $0.4 \mu$ pore diameter with $1.13 \mathrm{~cm}^{2}$ area. The inserts were thoroughly washed with $25 \mathrm{mM}$ HBSS Hank's balanced salt solution and $7.4 \mathrm{pH}$. The integrity of the monolayer formed was tested by monitoring Lucifer yellow dye permeability across the layer. Time dependent transport of BIC loaded MSNs was studied in unidirectional apical to basal manner. The donor compartment (apical) was treated with $0.5 \mathrm{~mL}$ of transport solution i.e. HBSS containing $0.1 \mathrm{mg} / \mathrm{mL}$ BIC and basal side was treated with $1.5 \mathrm{~mL}$ of HBSS solution. The samples were analysed by HPLC equipped with fluorescence detector with excitation wavelength of 260 and emission measured at $323 \mathrm{~nm}$.

After incubation of 30,60, 90, 120, 180, 240 and $300 \mathrm{~min}, 100 \mu \mathrm{L}$ aliquots were withdrawn from the receiver and replenished with same volume of fresh HBSS. The collected samples were further analysed by HPLC equipped with fluorescence detector. The apparent permeability coefficient (Papp) was measured using the following equation.

$$
\mathrm{P}_{\text {app }}=\frac{\mathrm{dQ}}{\mathrm{dt}} \times \frac{1}{\mathrm{AC}_{0}} \times 60
$$

Where,

Papp: Apparent permeability coefficient $(\mathrm{cm} / \mathrm{h})$

$\mathrm{dQ} / \mathrm{dt}$ : drug permeation rate $(\mathrm{mg} / \mathrm{min})$ 
A: cross-sectional area i.e. $1.13 \mathrm{~cm}^{2}$

$\mathrm{C}_{0}$ : Initial drug concentration in the donor compartment $(\mathrm{mg} / \mathrm{mL})$

\section{Pharmacokinetic study}

The study protocol to evaluate the pharmacokinetic parameters upon administration to SAM mice was approved by Institutional animal ethics committee (IAEC) constituted as per the guidelines of CPCSEA Committee for the purpose of control and supervision on experiments on animals, India. The protocol number was MSU/IAEC/2017-18/1724. About 4 months old healthy SAM were obtained from Zydus research centre, Ahmedabad, Gujarat. All the mice were given free access to food and water and acclimatized to the animal care facility for at least 7 days before starting the experiment.

To study the oral bioavailability, mice were assigned into 5 groups including control. The other groups were administered oral dose equivalent to $10 \mathrm{mg} / \mathrm{kg}$ BIC solution, BIC-MCM-41, BIC-MCM-41-AMN and MF by direct introduction into stomach using oral gavage.

About $0.3 \mathrm{~mL}$ of blood was collected from retro orbital vein of mice in EDTA containing centrifuge vials. Protein precipitation method was used in sample processing. Plasma was separated from the blood sample by centrifugation at $4000 \mathrm{rpm}$ for10 min at $4^{\circ} \mathrm{C}$. Processed sample was mixed with internal standard Tadalafil (TAD). Acetonitrile (ACN) was used as a precipitating agent. Supernatant was collected post centrifugation and quantified by HPLC equipped with a fluorescence detector. The developed HPLC method consisted of ammonium Formate $(20 \mathrm{mM})(\mathrm{pH} 4)$ and ACN in the ratio 46:54 run on a waters symmetry 300 $\mathrm{C}_{18}(250 \mathrm{~mm} \times 4.6 \mathrm{~mm} \times 5 \mu)$ column with a flow rate of $1 \mathrm{~mL} / \mathrm{min}$. Various Pharmacokinetic parameters were determined using an excel add-in.

\section{RESULTS}

\section{SEM and TEM analysis}

The synthesized silica nanoparticles were characterized by SEM-EDX and TEM. SEM was used to determine the morphological traits of MSNs. The SEM-EDX data was evident of successful functionalization with amino groups as seen in Figure 2. Elemental composition could be identified by the EDX data and was successfully correlated with bare and coated nature of silica nanoparticles. Post functionalization presence of nitrogen was observed as expected indicating successful aminopropyl functionalization. The porous order and nature of mesoporous nanoparticles was clearly visible
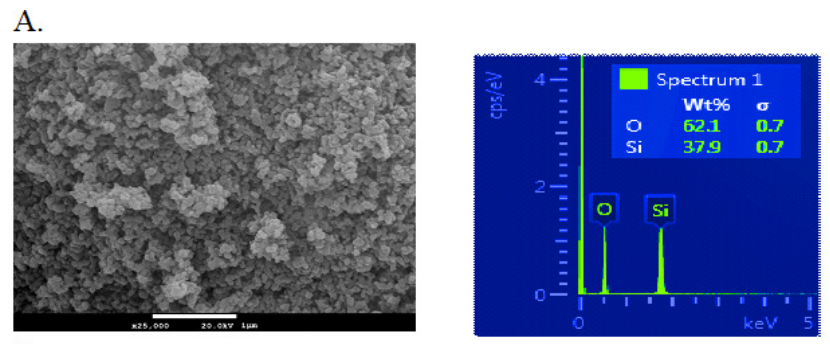

B.
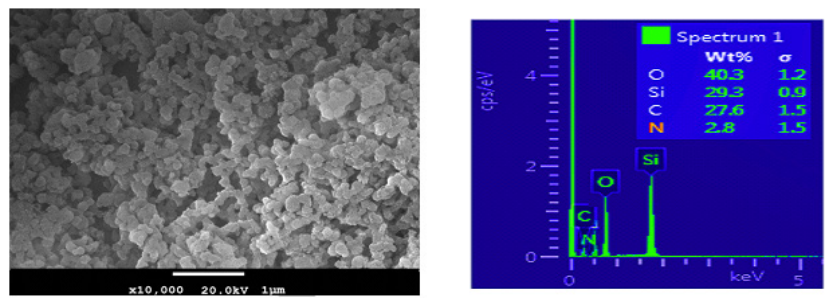

C.

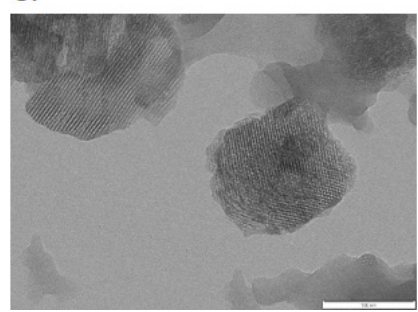

D.

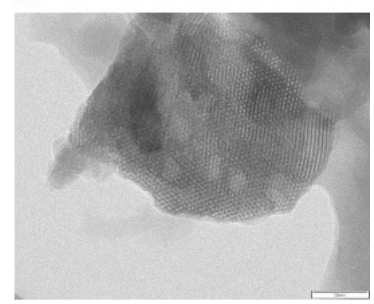

Figure 2: SEM-EDX data of A) MCM-41, B) MCM-41-AMN and TEM images of A) MCM-41, B) MCM-41-AMN.

in the images obtained. The honeycomb like cages formed within the materials are adhered to an amorphous silica wall periodically arranged on a lattice. ${ }^{16}$ TEM images depicted the almost spherical nature of synthesised particles preserved even after successful grafting of amino groups.

\section{FT-IR Spectroscopy}

FT-IR spectra provided proof of the manner in which successful synthesis and functionalization of MCM-41 mesoporous silica nanoparticles proceeded. The spectra of unfunctionalized MSNs were comparatively simple and major peaks could be easily assigned. In Figure 3, a characteristic peak attributed to BIC could be seen. The cyanide and amide carbonyl groups were denoted by peaks obtained at $2227 \mathrm{~cm}^{-1}, 1685 \mathrm{~cm}^{-1}$ and $3341 \mathrm{~cm}^{-1}$ respectively. Aromatic stretching due to phenyl group was observed at 708 and $833 \mathrm{~cm}^{-1}$. Characteristic cyano and carbonyl group peaks were seen at wavenumbers 2230 and $1689 \mathrm{~cm}^{-1}$ respectively. In case of nonfunctionalized MCM-41 (b) and (c) asynthesized and calcinated MCM-41 could be distinguished by nonexistence of C-H stretching (2954 and $2854 \mathrm{~cm}^{-1}$ ) and deformation vibrations at $1452 \mathrm{~cm}^{-1}$ In the spectra of calcinated MCM-41, stretching vibrations attributed to silanol groups gave a broad peak covering range 


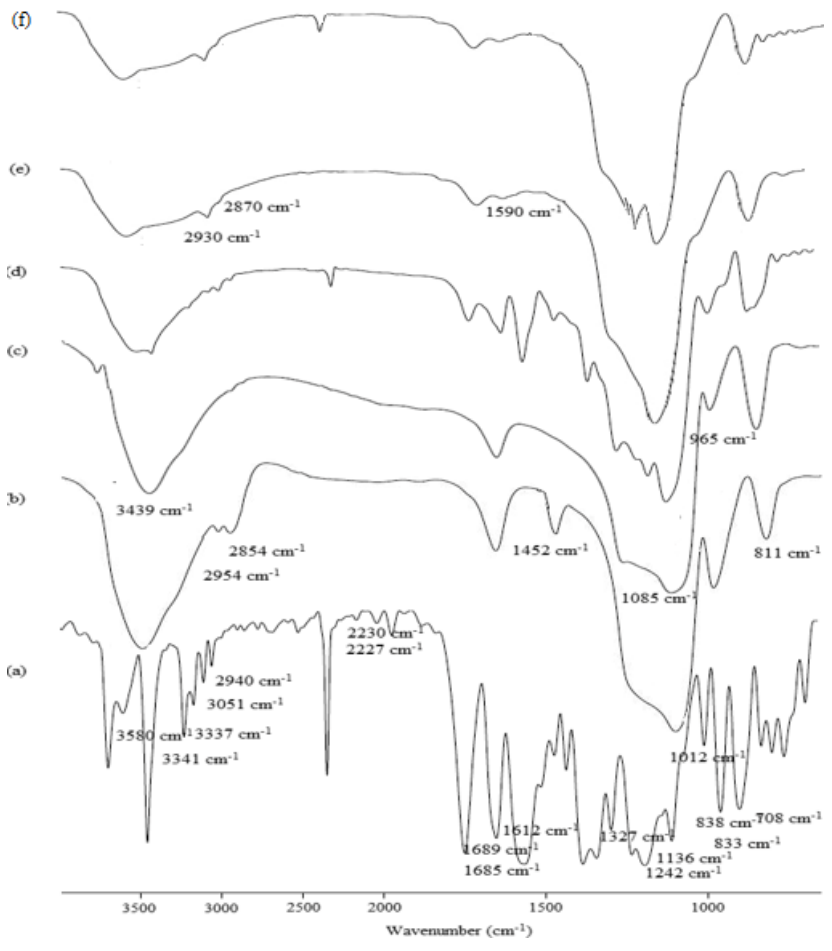

Figure 3: FT-IR Spectra (a) BIC (b) Asynthesized MSN (c) MCM-41 (d) BIC-MCM-41 (e)AMN-MSN (f) BIC-AMN-MSN.
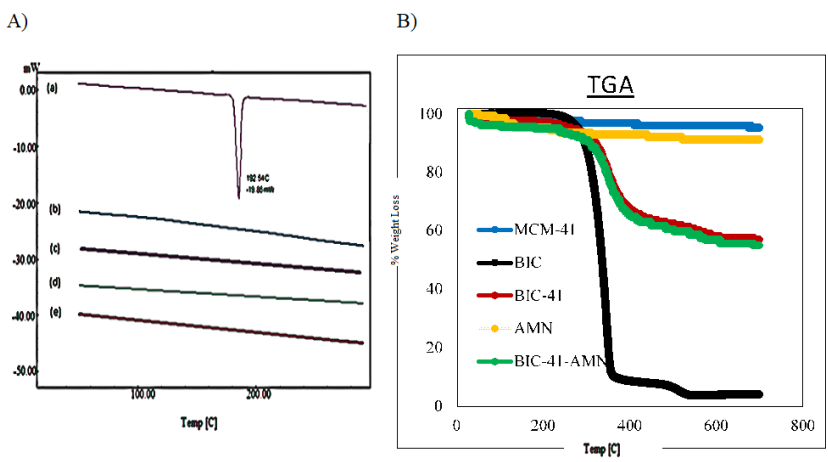

Figure 4: A) DSC and B) TGA analysis of Drug and BIC loaded MSNs.

of $3000-3750 \mathrm{~cm}^{-1}$. Bands observed at 811 and $1085 \mathrm{~cm}^{-1}$ were dedicated to Si-O bond stretching. Successful grafting of amino groups onto MCM-41 NPs was confirmed by a characteristic peak of asymmetric N-H bending vibration obtained at $1590 \mathrm{~cm}^{-1}$. This was further assisted by twin lets at 2930 and $2870 \mathrm{~cm}^{-1}$. Furthermore, complete encapsulation was also visible from the drug loaded mesoporous spectra.

\section{DSC and TGA analysis}

DSC was used in determining the melting point of the drug BIC and investigates complete entrapment at a preliminary level. The melting point of $\mathrm{BIC}$ was found to be $192.54^{\circ} \mathrm{C}$ denoted by a sharp endothermic peak.

\begin{tabular}{|c|c|c|c|c|c|}
\hline \multirow{2}{*}{$\begin{array}{l}\text { Sr } \\
\text { No. }\end{array}$} & \multirow{2}{*}{ Formulation } & \multirow{2}{*}{$\begin{array}{l}\text { D:C } \\
\text { Ratio }\end{array}$} & \multicolumn{2}{|c|}{$\%$ Loading } & \multirow{2}{*}{$\begin{array}{c}\% \\
\text { Entrapment }\end{array}$} \\
\hline & & & By TGA & By UV & \\
\hline 1. & BIC-MCM-41 & $1: 1.5$ & 38.95 & 39.12 & 96.48 \\
\hline 2. & $\begin{array}{l}\text { BIC-MCM-41- } \\
\text { AMN }\end{array}$ & 1:1.5 & 36.15 & 37.19 & 91.72 \\
\hline
\end{tabular}
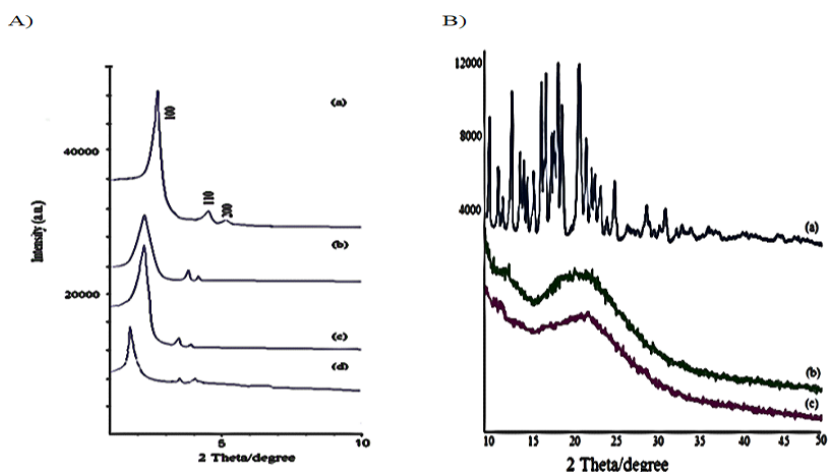

Figure 5: A) Small angle XRD (a) MCM-41 (b) BIC-MCM-41 (c) MCM-41-AMN (d) BIC-MCM-41-AMN and B) Wide angle XRD (a) BIC (b) BIC-MCM-41 (c) BIC-MCM-41-AMN.

The complete entrapment of drug into mesoporous carriers was confirmed by absence of any such peak in the thermogram (Figure 4A).

TGA analysis was useful in determining the $\%$ of grafting moieties on MCM-41 along with the \% drug loaded in both MCM-41 as well as MCM-41-AMN. Loss of surface silanol groups were the reason behind weight loss at higher temperature (Figure 4D). Decomposition of various groups was evident in range $150-600^{\circ} \mathrm{C}$. Although, a minor weight loss in the lower temperature reason could be credited to the surface dehydration by loss of water molecules. As calculated from the thermogram $\%$ grafting of amino groups was found to be $4.0 \%$. The $\%$ of BIC loaded into MCM- 41 was MCM41-AMN was 38.95 and $36.15 \%$ respectively as estimated by TGA. UV estimation gave a \% loading of 39.12 and 37.19 respectively for MCM-41 and MCM-41-AMN (Table 3).

\section{X-RAY diffraction studies}

Small angle and wide-angle X-Ray diffraction studies were carried out to ensure the integrity of mesoporous skeleton as well preserved and transition of nature of drug. In small angle XRD spectra three peaks characteristic to MCM-41 mesoporous carriers were obtained at 100, 110 and 200 reflection peaks. ${ }^{17}$ 
This pattern was well preserved and even after functionalization and drug loading as seen from the Figure 5A.

Crystalline nature of BIC was justified by the sharp and highly intensified peaks obtained in the wide angle XRD spectra. The position of the peaks identified in order of their ascending theta values are 12.29, 18.15, 23.29, $24.11,29.1,29.30,31.45$ and $34.82 \theta$. When we see the Wide-angle spectra of drug loaded MSNs, no clear intense peak is visible (Figure 5B). This indicates the conversion of crystalline BIC to amorphous form after entrapment into MSNs. This fact could play a major part in dissolution enhancement of drug.

\section{Zeta potential and size}

Zeta potential was useful in determining surface residual charge. Presence of huge number of silanol groups by silanol deprotonation are responsible for negative zeta potential of $-36.86 \mathrm{mV}$ for $\mathrm{MCM}-41 .^{18}$ Post functionalization with aminopropyl groups gave a positive zeta potential value of $+33.93 \mathrm{mV}$ attributed to large number of positively charged amino groups. Notably, post drug loading into MCM-41 and MCM41-AMN gave a zeta potential of -24.74 and $+36.98 \mathrm{mV}$. Zetasizer gave the $\mathrm{z}$ average diameter and PDI of synthesized nanoparticles. The $\mathrm{z}$ average diameter for MCM-41 and MCM-41-AMN was found to be $110.2 \mathrm{~nm}$ and $124.7 \mathrm{~nm}$. The PDI value for bare and functionalized MSNs was 0.34 and 0.28 respectively (Figure 6). Hence, as expected here was an increase in particle size with functionalization due to addition of bulky group on the surface.

\section{Nitrogen adsorption-desorption studies (Porosity and surface areadetermination)}

The adsorption-desorption isotherms obtained were having well defined capillary condensation step exhibiting type 4 characteristics. This was evident of mesopores being homogenous in size. ${ }^{19}$ Highest surface area and pore size was observed for MCM-41 mesoporous carrier. Amine functionalized mesoporous carrier displayed decrease in the porosity and surface area as compared to their bare counterparts. Similarly, both the properties also decreased when drug was loaded into the mesopores due to the pores being occupied by drug molecules. The results are summarized in Table 4 and Figure 7.

\section{In vitro dissolution Kinetics}

Drug release study was carried out in $1000 \mathrm{~mL}$ water with $0.5 \%$ SLS to determine the release pattern by
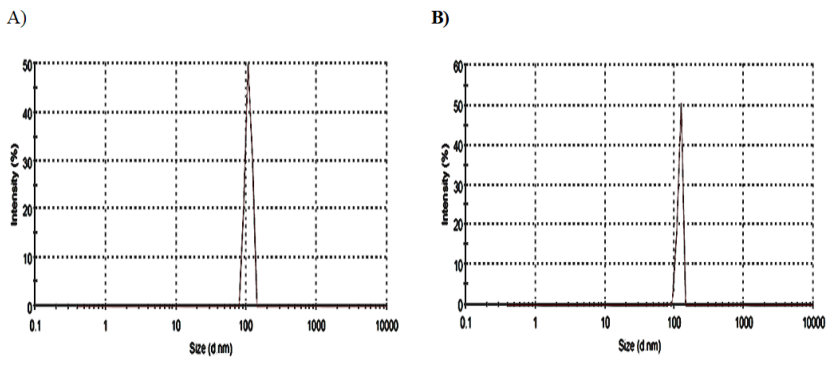

Figure 6: Size distribution by intensity ( $Z$ average $110.2 \mathrm{~nm}$ ) and PDI 0.34 for (a) MCM-41 and (b) MCM-41-AMN ( $Z$ average 124.7 $\mathrm{nm}$ ) and PDI 0.28.

A)
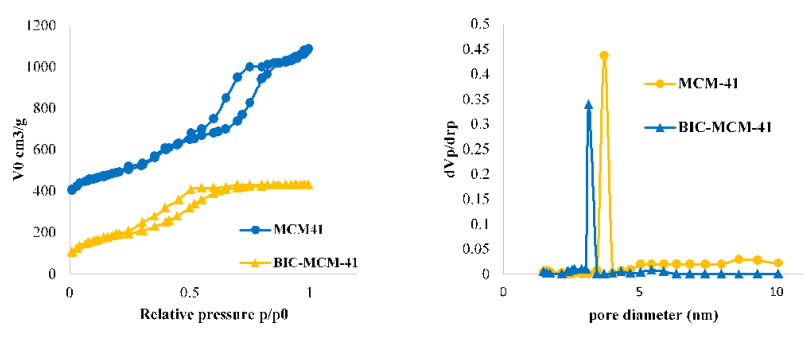

B)
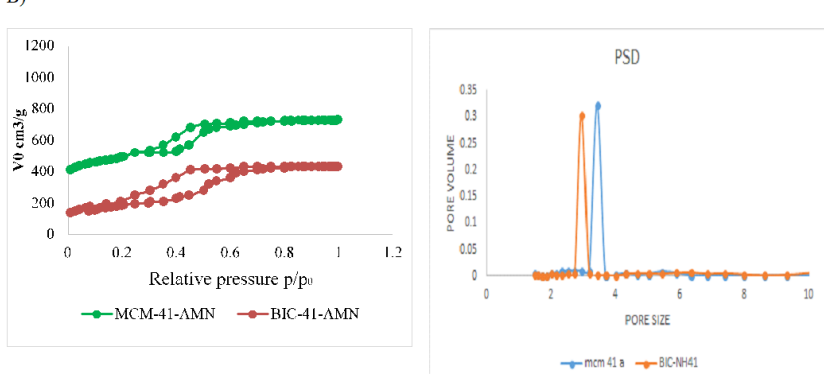

Figure 7: A) Surface area and pore size distribution of MCM-41 and B) MCM-41-MSN.

\begin{tabular}{|c|c|c|c|c|}
\hline \multicolumn{5}{|c|}{ Table 4: Surface area and porosity data for } \\
mesoporous nanoparticles (Bare and drug loaded). \\
\hline $\begin{array}{c}\text { SAMPLE } \\
\text { NAME }\end{array}$ & $\begin{array}{c}\text { BET } \\
\text { Surface } \\
\text { area }\left(\mathbf{m}^{2} / \mathbf{g}\right)\end{array}$ & $\begin{array}{c}\text { BJH } \\
\text { surface } \\
\text { area }\left(\mathbf{m}^{2} / \mathbf{g}\right)\end{array}$ & $\begin{array}{c}\text { Pore } \\
\text { size } \\
\text { (nm) }\end{array}$ & $\begin{array}{c}\text { Pore } \\
\text { volume } \\
\left.\mathbf{( c m}^{3} / \mathbf{g}\right)\end{array}$ \\
\hline MCM-41 & 1089.97 & 1441.97 & 3.69 & 0.4377 \\
\hline $\begin{array}{c}\text { MCM-41- } \\
\text { AMN }\end{array}$ & 827.90 & 1165.49 & 3.43 & 0.4261 \\
\hline $\begin{array}{c}\text { BIC- } \\
\text { MCM-41 }\end{array}$ & 813.28 & 902.26 & 3.13 & 0.3397 \\
\hline $\begin{array}{c}\text { BIC-MCM- } \\
41-A M N\end{array}$ & 762.12 & 798.04 & 2.91 & 0.3041 \\
\hline
\end{tabular}


calculating the cumulative drug release at different time points. BIC release from MCM-41 was highest followed by marketed formulation. However, a controlled and sustained behaviour of drug release was obtained in case of amine functionalized MSNs. Additionally, fast and fed state gastric and intestinal media were used to study if there is any effect of food on drug release from all the formulations..$^{20}$ The drug release was almost similar in case of both fast and fed state conditions and hence it could be concluded that presence of food did not alter drug release (Figure 8). Thus, the medication could be taken either ways empty or after meal, it wouldn't affect the absorption or release. Further, various dissolution kinetic models were applied on the obtained \%CDR data. Of the various models applied he highest regression coefficient and MSC value along with lowest AIC criteria was best fit in Weibull model in case of MCM-41 type mesoporous matrix ${ }^{21}$ (Table 5). Whereas, in case of MCM-41-AMN matrix Higuchi model was found to the best fit satisfying all the three criteria (Table 6). Best fit models were understandably different for both release systems as a different release
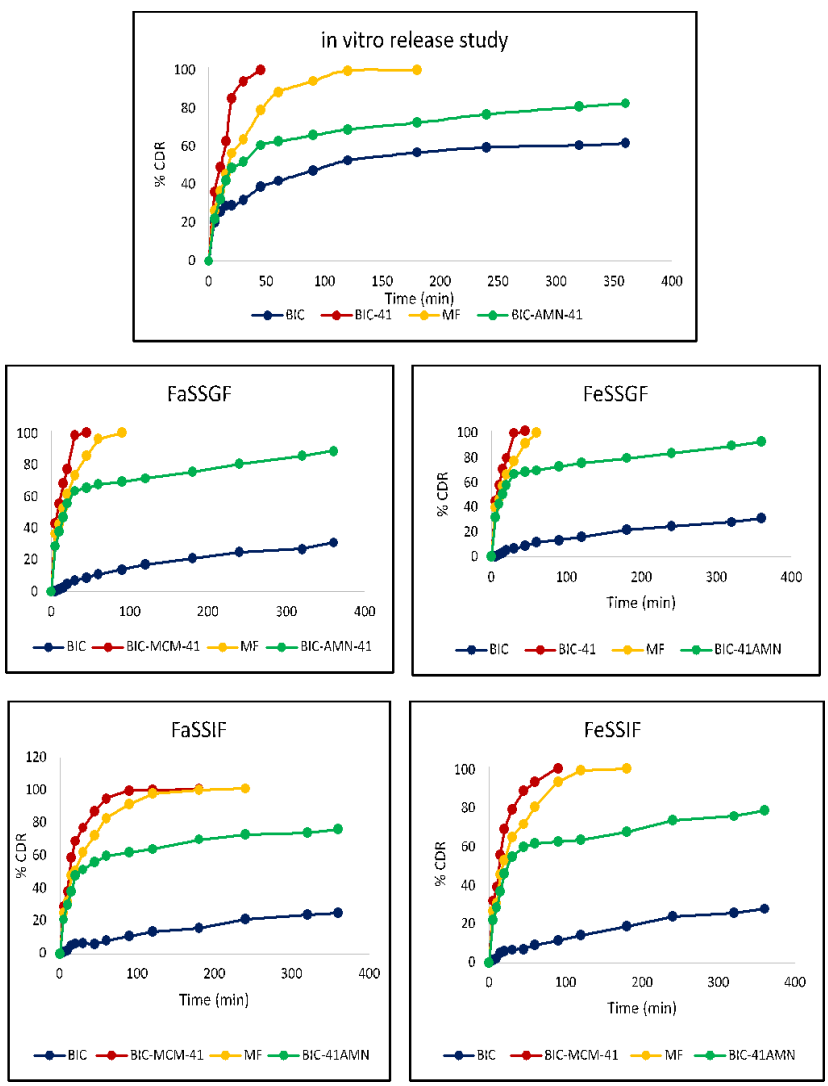

Figure 8: In vitro drug release study in various media. pattern was seen in both. MCM-41 gave an initial burst release and faster one as compared to somewhat slower and sustained release action as observed in aminated matrix.

\section{MTT assay results}

The toxicity of synthesized nanoparticles was compared on Caco-2 cell lines. The study was conducted in the concentration range $0.1-100 \mu \mathrm{g} / \mathrm{mL}$ (Figure 9). Comparison of cytotoxicity was done amongst BIC, BIC-MCM-41 and BIC-MCM-AMN-41 NPs. The MT'T assay was performed providing an incubation time of $4 \mathrm{~h}$. The results indicated relatively safer nature of MSNs on Caco-2 cells in the concentration range of $10-100 \mu \mathrm{g} / \mathrm{mL}$. The percentage viability was found to be greater than 90 percent in all cases.

\section{Caco-2 monolayer Permeability}

From the results obtained in cell cytotoxicity assay concentration of $100 \mu \mathrm{g} / \mathrm{mL}$ was selected to be safe for proceeding further with cell permeability study. Experiment was conducted for BIC, BIC loaded MCM-41 and BIC-MCM-AMN-41 from apical to basal compartment. Papp value was estimated for various formulations (Table 7). There was an improvement of 4.66-fold in permeability of with respect. Therefore, it could be concluded that BIC encapsulation into the mesoporous network proved to be beneficial in enhancing its permeability as well.

\section{In vitro pharmacokinetic studies}

Poor bioavailability of BIC is attributed to its limited solubility. To determine the oral bioavailability of BIC pharmacokinetic study of BIC, BIC-MCM-41 and BICMCM-41-AMN was carried out in male swiss albino mice (Figure 10). All the blood samples collected were adequately processed and analysed by a well-developed and validated RP-HPLC-FL method. Different pharmacokinetic parameters like $t_{1 / 2}$, AUC etc were calculated for administered samples and the results are summarized in Table 8 . The outcome gave a significant increment in drug plasma concentration $(p<0.05)$ on administration of formulated nanoparticle as compared to drug alone. $\mathrm{C}_{\max }$ was highest for BIC-MCM-41 NPs. Highest bioavailability and AUC were obtained using MCM-41 carriers. The BA of BIC-MCM-41 MSNs was 2.61 and 1.38 times more as compared to BIC and MF respectively. Whereas, in case of functionalized matrix the BA was enhanced 1.71 times the drug solution. 


\begin{tabular}{|c|c|c|c|c|c|c|c|c|}
\hline \multirow[t]{2}{*}{ Formulation } & \multirow{2}{*}{$\begin{array}{l}\text { Statistical } \\
\text { Parameters }\end{array}$} & \multirow[t]{2}{*}{ Dissolution media } & \multicolumn{6}{|c|}{ Dissolution model } \\
\hline & & & Zero & First & Weibull & Higuchi & $\begin{array}{c}\text { Hixon } \\
\text { Crowell }\end{array}$ & $\begin{array}{c}\text { Korsmeyer } \\
\text { peppas }\end{array}$ \\
\hline \multirow[t]{5}{*}{ BIC-MCM-41 } & \multirow[t]{5}{*}{$\mathrm{R}^{2}$} & $0.5 \%$ SLS in water & 0.8898 & 0.9894 & 0.9900 & 0.9585 & 0.9840 & 0.9804 \\
\hline & & $0.5 \%$ SLS in FaSSGF & 0.5206 & 0.9796 & 0.9848 & 0.9672 & 0.9720 & 0.9813 \\
\hline & & $0.5 \%$ SLS in FeSSGF & 0.4807 & 0.9799 & 0.9862 & 0.9615 & 0.9707 & 0.9819 \\
\hline & & $0.5 \%$ SLS in FaSSIF & 0.0139 & 0.9911 & 0.9936 & 0.8449 & 0.9403 & 0.9476 \\
\hline & & $0.5 \%$ SLS in FeSSIF & -0.0086 & 0.9907 & 0.9934 & 0.8426 & 0.9384 & 0.9521 \\
\hline \multirow[t]{5}{*}{ BIC-MCM-41 } & \multirow[t]{5}{*}{ AIC } & $0.5 \%$ SLS in water & 69.43 & 41.25 & 38.00 & 48.89 & 41.55 & 44.88 \\
\hline & & $0.5 \%$ SLS in FaSSGF & 59.21 & 37.11 & 37.07 & 40.44 & 39.33 & 40.52 \\
\hline & & $0.5 \%$ SLS in FeSSGF & 59.88 & 37.13 & 36.46 & 41.67 & 39.75 & 40.39 \\
\hline & & $0.5 \%$ SLS in FaSSIF & 94.22 & 47.17 & 47.82 & 75.72 & 66.18 & 66.88 \\
\hline & & $0.5 \%$ SLS in FeSSIF & 94.45 & 47.59 & 48.17 & 75.88 & 66.50 & 65.99 \\
\hline \multirow[t]{5}{*}{ BIC-MCM-41 } & \multirow[t]{5}{*}{ MSC } & $0.5 \%$ SLS in water & -0.2918 & 3.19 & 3.63 & 2.27 & 3.23 & 2.77 \\
\hline & & $0.5 \%$ SLS in FaSSGF & -0.33 & 2.71 & 2.82 & 2.34 & 2.50 & 2.33 \\
\hline & & $0.5 \%$ SLS in FeSSGF & -0.45 & 2.79 & 2.89 & 2.14 & 2.42 & 2.32 \\
\hline & & $0.5 \%$ SLS in FaSSIF & -0.72 & 3.98 & 3.91 & 1.12 & 2.08 & 2.01 \\
\hline & & $0.5 \%$ SLS in FeSSIF & -0.75 & 3.92 & 3.86 & 1.09 & 2.03 & 2.08 \\
\hline
\end{tabular}

\begin{tabular}{|c|c|c|c|c|c|c|c|c|}
\hline \multirow[t]{2}{*}{ Formulation } & \multirow{2}{*}{$\begin{array}{c}\text { Statistical } \\
\text { Parameters }\end{array}$} & \multirow[t]{2}{*}{ Dissolution media } & \multicolumn{6}{|c|}{ Dissolution model } \\
\hline & & & Zero & First & Weibull & Higuchi & $\begin{array}{l}\text { Hixon } \\
\text { Crowell }\end{array}$ & $\begin{array}{c}\text { Korsmeyer } \\
\text { peppas }\end{array}$ \\
\hline \multirow[t]{5}{*}{ BIC-AMN-41 } & \multirow[t]{5}{*}{$\mathrm{R}^{2}$} & $0.5 \%$ SLS in water & -1.0709 & 0.4828 & 0.3995 & 0.9868 & 0.2041 & 0.9475 \\
\hline & & $0.5 \%$ SLS in FaSSGF & -1.3167 & 0.5656 & 0.2959 & 0.9853 & 0.1588 & 0.9585 \\
\hline & & $0.5 \%$ SLS in FeSSGF & -1.4610 & 0.6499 & 0.2376 & 0.9893 & 0.1417 & 0.9681 \\
\hline & & $0.5 \%$ SLS in FaSSIF & -1.003 & 0.3247 & 0.4367 & 0.9892 & 0.1023 & 0.9517 \\
\hline & & $0.5 \%$ SLS in FeSSIF & -0.9471 & 0.3854 & 0.4502 & 0.9786 & 0.1563 & 0.9427 \\
\hline \multirow[t]{5}{*}{ BIC-AMN-41 } & \multirow[t]{5}{*}{ AIC } & $0.5 \%$ SLS in water & 136.90 & 117.47 & 119.56 & 70.14 & 123.51 & 87.46 \\
\hline & & $0.5 \%$ SLS in FaSSGF & 139.19 & 115.76 & 122.52 & 72.37 & 125.01 & 84.88 \\
\hline & & $0.5 \%$ SLS in FeSSGF & 140.77 & 113.47 & 124.37 & 68.62 & 126.02 & 81.93 \\
\hline & & $0.5 \%$ SLS in FaSSIF & 134.62 & 119.40 & 116.86 & 65.47 & 123.39 & 85.64 \\
\hline & & $0.5 \%$ SLS in FeSSIF & 135.03 & 118.89 & 117.33 & 75.88 & 123.32 & 87.67 \\
\hline \multirow[t]{5}{*}{ BIC-AMN-41 } & \multirow[t]{5}{*}{ MSC } & $0.5 \%$ SLS in water & -1.41 & -0.026 & -0.17 & 3.35 & -0.45 & 2.11 \\
\hline & & $0.5 \%$ SLS in FaSSGF & -1.60 & 0.072 & -0.41 & 3.17 & -0.58 & 2.27 \\
\hline & & $0.5 \%$ SLS in FeSSGF & -1.70 & 0.24 & -0.53 & 3.44 & -0.65 & 2.49 \\
\hline & & $0.5 \%$ SLS in FaSSIF & -1.36 & -0.27 & -0.09 & 3.57 & -0.55 & 2.22 \\
\hline & & $0.5 \%$ SLS in FeSSIF & -1.31 & -0.16 & -0.05 & 2.90 & -0.48 & 2.06 \\
\hline
\end{tabular}




\begin{tabular}{|c|c|c|c|}
\hline \multicolumn{4}{|c|}{ PERMEABILITY $\left(P_{\text {app }}\right)(\mathrm{cm} / \mathrm{s})$} \\
\hline TIME (min) & BIC & BIC 41 & BIC-NH2-41 \\
\hline 30 & $0.65 \pm 0.04$ & $4.96 \pm 0.72$ & $3.56 \pm 0.51$ \\
\hline 60 & $1.52 \pm 0.22$ & $15.67 \pm 1.26$ & $9.93 \pm 1.38$ \\
\hline 90 & $3.81 \pm 0.56$ & $25.49 \pm 1.89$ & $13.32 \pm 1.66$ \\
\hline 120 & $5.93 \pm 0.87$ & $32.88 \pm 2.04$ & $19.57 \pm 2.17$ \\
\hline 180 & $6.99 \pm 1.07$ & $38.15 \pm 2.98$ & $25.24 \pm 2.56$ \\
\hline 240 & $9.82 \pm 1.39$ & $43.07 \pm 3.01$ & $30.08 \pm 2.82$ \\
\hline 300 & $11.12 \pm 1.84$ & $49.83 \pm 3.47$ & $31.82 \pm 3.05$ \\
\hline Papp & $1.96^{*} 10^{-2}$ & $9.14^{*} 10^{-2}$ & $5.31^{*} 10^{-2}$ \\
\hline
\end{tabular}

\begin{tabular}{|c|c|c|c|c|c|}
\hline $\begin{array}{l}\text { PARAMETERS } \\
\text { SAMPLE }\end{array}$ & $T_{1 / 2}$ & $\mathbf{T}_{\text {max }}$ & $C_{\max }$ & $\begin{array}{c}\text { AUC } \\
\text { 0-inf_obs }\end{array}$ & $\begin{array}{l}\text { MRT } \\
\text { 0-inf_obs }\end{array}$ \\
\hline BICALUTAMIDE & 12.55 & 8 & 3.24 & 76.58 & 20.89 \\
\hline BIC-41 & 13.61 & 2 & 9.88 & 199.23 & 20.55 \\
\hline BIC-AMN-41 & 12.38 & 4 & 6.43 & 130.99 & 19.21 \\
\hline $\begin{array}{l}\text { MARKETED } \\
\text { FORMULATION }\end{array}$ & 11.66 & 2 & 8.32 & 144.26 & 17.61 \\
\hline
\end{tabular}

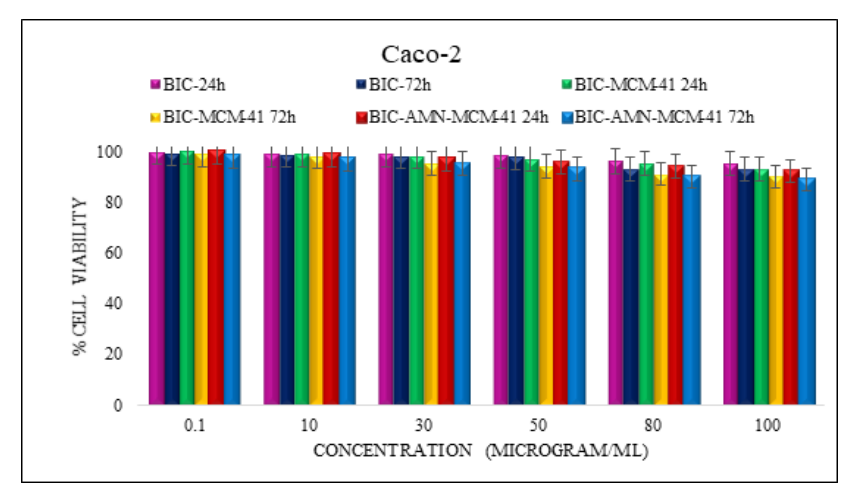

Figure 9: MTT assay results of Caco-2 cells.
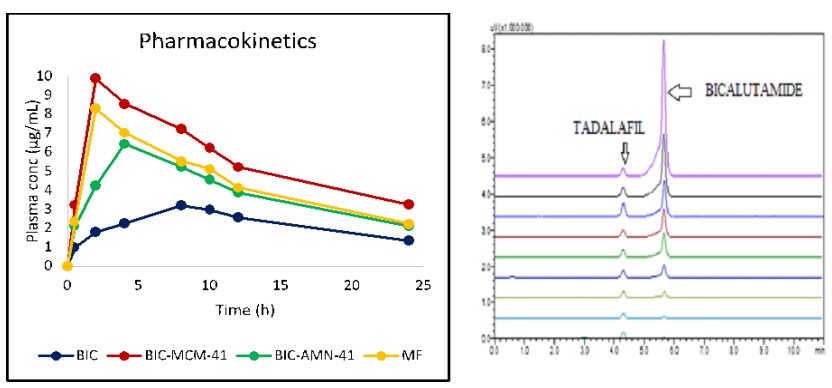

Figure 10: Oral Pharmacokinetic data and chromatogram of drug Bicalutamide with Internal standard Tadalafil.

\section{DISCUSSION}

Though various other nano drug delivery systems have been explored for this purpose. Use of MSNs for BA enhancement of BC is not reported so far. A relatively facile procedure was used for MSN synthesis and functionalization of MCM-41 type mesoporous nanocarriers. The aim of the entire research was to study the use of MSNs and determine how far they are useful in enhancing the dissolution rate and solubility of BCS Class II drug BIC. The synthesized nanoparticles were subjected to in-depth characterization using various techniques. SEM-EDX data and TEM images were proof of mesoporous nature of synthesized nanomaterial and successful introduction of amino groups using APTES, this was seen by introduction of element $\mathrm{N}$ in the EDX data $\mathrm{f}$ MCM-41-AMN. FT-IR spectra were neat enough in depicting the characteristic functional groups and various stretching and bending vibrations clearly. Additionally, successful loading of drug was confirmed by DSC thermogram and XRD spectra. The loading ratio of drug and carrier was optimized as 1:1.5. The \% loading calculated by UV and TGA were almost similar and high loading and entrapment efficiency was obtained.

The wide angle XRD spectra for drug contained sharps well defined peaks with significant intensities. However, these were different in case of drug loaded MSNs. This could explain the possibility of conversion of crystalline nature of drug to amorphous. The increment in dissolution rate could be explained by this possible change in nature of drug after encapsulation into mesopores. A low PDI value indicated a uniform sized population of nanoparticles. Also, zeta potential was useful in determining the successful functionalization and drug loading at each step. The pore size, pore volume and surface area all decreased as functionalization proceeded further. Similar trend was observed post drug loading. Understandably due to increase in the number of occupied pores.

In vitro drug dissolution studies and kinetic modelling gave some useful results. A characteristic burst release pattern was observed in case of drug loaded MCM-41 NPs. Comparatively, a slow release was seen in amine functionalized mesoporous nanoparticles. Thus, the best fit models were also different in both the cases. In case of MCM-41, Weibull model was found to be the best fit one. Also, for MCM-41-AMN Higuchi model was the best fit one. Linear relationship between the fraction of release of ETO and square root of time, suggested a Higuchi diffusion process. This was again 
complementary to the AIC and MSC values obtained for Higuchi model.

The MTT assay performed on Caco-2 cell line was useful in determining the safe concentration of NPs which could be used for permeability studies. Caco-2 monolayer permeability suggested an increased permeability of the synthesized drug loaded MSNs as compared to plain drug. The pharmacokinetic data evaluation exhibited an improved bioavailability as well for the mesoporous nanoparticles. Thus, MSNs were found to be useful in enhancing the dissolution rate, permeability and ultimately the bioavailability of the developed drug loaded nanoparticles.

\section{CONCLUSION}

Both the synthesized MSNs proved to be efficient vehicles for BIC delivery. The identical drug release study performed in fast and fed state dissolution media suggested that food intake did not have a significant impact on food absorption. The significant improvement in the dissolution rate could be attributed to the change of crystalline form of drug to amorphous form. Amination played a capping role and somewhat hindered the drug release leading to a sustained drug delivery. There was also a significant increase in permeability in case of mesoporous formulation. Lastly, positive outcome was obtained and 2.61 times increment in bioavailability was obtained. These all results combined together suggested that MSNs hold a huge potential as drug delivery carriers and can be useful in wide applications like dissolution, permeability and bioavailability enhancement.

\section{ACKNOWLEDGEMENT}

The author would like to acknowledge the Maharaja Sayajirao University of Baroda for providing financial support and adequate platform to carry out the research work.

\section{CONFLICT OF INTEREST}

The authors declare that there is no conflict of interest.

\section{ABBREVIATIONS}

ACN: Acetonitrile; API: Active pharmaceutical ingredient; APTES: (3-Aminopropyl) triethoxy silane; BIC: Bicalutamide; CTAB: Cetyltrimethylammonium Bromide; DLS: Dynamiclightscattering; DMEM: Dulbecco's Modified Eagle Medium; DSC: Differential
Scanning Calorimetry; FaSSGF: Fasted State Simulated Gastric Fluid; FaSSIF: Fasted State Simulated Intestinal Fluid; FeSSGF: Fed State Simulated Gastric Fluid; FeSSIF: Fed State Simulated Intestinal Fluid; GRAS: Generally Regarded As Safe; HBSS: Hank's balanced salt solution; IAEC: Institutional animal ethics committee; MCM: Mobile Composition Of Matter; MSNs: Mesoporous Silica Nanoparticles; MTT: 3-(4,5-dimethylthiazol-2-yl)-2,5-diphenyl tetrazolium bromide; NCCS: National centre for cell sciences; RBF: Round bottom flask; SAM: Swiss Albino Mice; SEM: Scanning Electron Microscopy; TAD: Tadalafil; TEM: Transmission Electron Microscopy; TGA: Thermo Gravimetric Analysis; TMAOH: Tetramethyl ammonium hydroxide pentahydrate; XRD: X-Ray Diffraction.

\section{REFERENCES}

1. Kalepu S, Nekkanti V. Insoluble drug delivery strategies: Review of recent advances and business prospects. Acta Pharmaceutica Sinica B. 2015;5(5):442-53.

2. Joyce R, Fenton MA, Rode P, Constantine M, Gaynes L, Kolvenbag G, et al. High dose bicalutamide for androgen independent prostate cancer: Effect of prior hormonal therapy. The Journal of Urology. 1998;159(1):149-53.

3. Pokharkar VB, Malhi T, Mandpe L. Bicalutamide nanocrystals with improved oral bioavailability: In vitro and in vivo evaluation. Pharmaceutical Development and Technology. 2013;18(3):660-6.

4. Ren F, Jing Q, Tang Y, Shen Y, Chen J, Gao F, et al. Characteristics of bicalutamide solid dispersions and improvement of the dissolution. Drug Development and Industrial Pharmacy. 2006;32(8):967-72.

5. Sancheti P, Vyas V, Shah M, Karekar P, Pore Y. Development and characterization of bicalutamide-poloxamer F68 solid dispersion systems. Die Pharmazie-An International Journal of Pharmaceutical Sciences. 2008;63(8):571-5.

6. Li C, Le Y, Chen JF. Formation of bicalutamide nanodispersion for dissolution rate enhancement. International Journal of Pharmaceutics. 2011;404(1-2): 257-63.

7. Vaniya BK, Shah SK, Patel DJ, Khatri HN. Physicochemical Characterization and in-vitro Dissolution Enhancement of Bicalutamide-Hp-B-Cd Complex. Journal of Pharmaceutics and Drug Delivery Research. 2017;3(2).

8. Srikanth M, Ram BJ, Rajan DS, Adinarayana G, Murthy KR. Dissolution rate enhancement of bicalutamide by adsorption process. African Journal of Pharmacy and Pharmacology. 2013;7(21):1357-62.

9. Chen H, Zhen Z, Tang W, Todd T, Chuang YJ, Wang L, et al. Label-free luminescent mesoporous silica nanoparticles for imaging and drug delivery. Theranostics. 2013;3(9):650.

10. Watermann A, Brieger J. Mesoporous silica nanoparticles as drug delivery vehicles in cancer. Nanomaterials. 2017;7(7):189.

11. Bharti C, Nagaich U, Pal AK, Gulati N. Mesoporous silica nanoparticles in target drug delivery system: A review. International Journal of Pharmaceutical Investigation. 2015;5(3):124.

12. Fan J, Fang G, Wang X, Zeng F, Xiang $\mathrm{Y}$, Wu S. Targeted anticancer prodrug with mesoporous silica nanoparticles as vehicles. Nanotechnology. 2011;22(45):455102.

13. Saroj S, Rajput SJ. Composite smart mesoporous silica nanoparticles as promising theranostic candidates: Recent trends and applications. Journal of Drug Delivery Science and Technology. 2018;44:349-65. 
14. Wouters BH, Chen T, Dewilde M, Grobet PJ. Reactivity of the surface hydroxyl groups of MCM-41 towards silylation with trimethylchlorosilane. Microporous and Mesoporous Materials. 2001;44:453-7.

15. Yoncheva K, Popova M, Szegedi A, Mihály J, Tzankov B, Lambov N, et al. Functionalized mesoporous silica nanoparticles for oral delivery of budesonide. Journal of Solid State Chemistry. 2014;211:154-61.

16. Rades S, Hodoroaba VD, Salge T, Wirth T, Lobera MP, Labrador RH, et al. High-resolution imaging with SEM/T-SEM, EDX and SAM as a combined methodical approach for morphological and elemental analyses of single engineered nanoparticles. RSC Advances. 2014;4(91):49577-87.

17. Sun JT, Hong CY, Pan CY. Fabrication of PDEAEMA-coated mesoporous silica nanoparticles and $\mathrm{pH}$-responsive controlled release. The Journal of Physical Chemistry C. 2010;114(29):12481-6.
18. Knežević NŽ, Durand JO. Targeted treatment of cancer with nanotherapeutics based on mesoporous silica nanoparticles. Chem Plus Chem. 2015;80(1):26-36.

19. Kachbouri S, Mnasri N, Elaloui E, Moussaoui Y. Tuning particle morphology of mesoporous silica nanoparticles for adsorption of dyes from aqueous solution. Journal of Saudi Chemical Society. 2018;22(4):405-15.

20. Shah PV, Rajput SJ. A comparative in vitro release study of raloxifene encapsulated ordered MCM-41 and MCM-48 nanoparticles: A dissolution kinetics study in simulated and biorelevant media. Journal of Drug Delivery Science and Technology. 2017;41:31-44.

21. Shah P, Rajput SJ. Amine decorated $2 d$ hexagonal and $3 d$ cubic mesoporous silica nanoparticles: A comprehensive dissolution kinetic study in simulated and biorelevant media. Journal of Dispersion Science and Technology. 2018;1:1-19.

\section{PICTORIAL ABSTRACT}

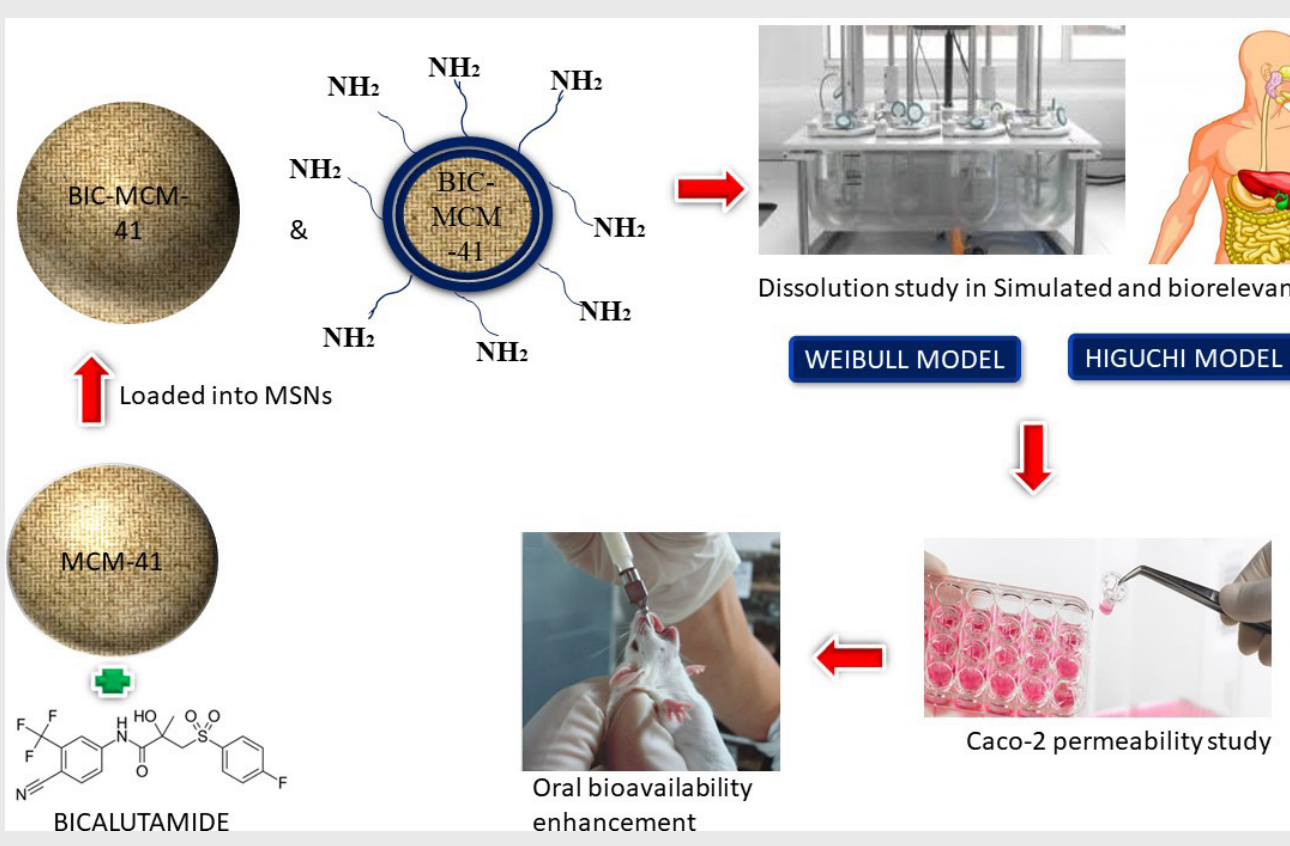

\section{SUMMARY}

The research outcome suggested a pivotal role of MSNs as drug delivery systems in increasing the dissolution and bioavailability of anticancer drug Bicalutamide. Both bare and surface functionalised MSNs, which were given amine coating proved to be good potential delivery agents. Amine functionalisation was responsible for modified delivery. Drug loaded bare MSNs led to enhanced dissolution rate of BIC which has good potential in cancer treatment; which to some extent is marred by its limited solubility. Dissolution kinetics study revealed Weibull and Higuchi to be the best fit model for MCM-41 and MCM-41-AMN carriers. Further, ultimate goal of good enhancement in bioavailability was also achieved as seen in results from the oral pharmacokinetic study conducted on mice. It can be concluded that MSNs served as excellent nanocarriers for model drug BIC aiding in its dissolution, permeability and bioavailability enhancement. They can be ideal rescuers for drugs whose efficacy is limited by solubility and permeability. Though their efficacy and safety in human clinical trials remains to be established, since silica is regarded as GRAS by USFDA, the hope is still alive for establishing them as safe and effective carriers. 


\section{About Authors}

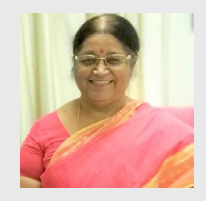

Sadhana Rajput is a Professor and Dean in Pharmaceutical Quality Assurance Department at Faculty of Pharmacy, The Maharaja Sayajirao University of Baroda. Her area of interests is Development of analytical methods, Impurity profiling, Development of modified release formulations and standardization of

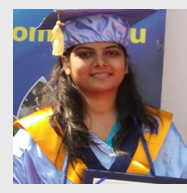

Seema Saroj has completed her Ph.D. in Nano drug delivery- Mesoporous silica nanoparticles from Faculty of Pharmacy, The Maharaja Sayajirao University of Baroda. She has completed her M.S.Pharm from NIPER-Ahmedabad. She is currently working in formulation development department on developing herbal formulations. (Peptides) at Omgene (Peptides) at Omgene Lifesciences Pvt Ltd, Vadodara.

Cite this article: Saroj S, Rajput S. Bioavailability and Dissolution Rate Enhancement of an Anticancer Drug Bicalutamide by Encapsulation into Mesoporous Silica Nanoparticles: Effects of Amine Functionalization and Caco-2 Cell Permeability Study. Indian J of Pharmaceutical Education and Research. 2020;54(3):589-601. 